\title{
La Higiene en el currículo escolar de la Educación Obligatoria española
}

\section{The Hygiene in the Spanish official curriculum of the Compulsory Education}

\section{Lucía Fernández-Manzano; Marta Talavera Carles Furió; Valentín Gavidia}

Dpto. Didáctica CC. Experimentales y Sociales Universidad de Valencia

Resumen: El objetivo del presente trabajo ha sido doble: determinar las competencias en Higiene que deben adquirir los jóvenes españoles en su paso por la educación obligatoria y analizar la contribución que hacen los distintos currículos oficiales publicados, al amparo de la Ley Orgánica de Educación (LOE, 2006), en el BOE y en el DOCV en el desarrollo de las citadas competencias. Con esta finalidad, se ha elaborado un Instrumento de análisis que recoge nueve problemas de salud y las competencias a adquirir por el alumnado de la educación obligatoria para hacerles frente en el ámbito de la Higiene. Para su confección, se efectuó un estudio Delphi y una reunión presencial de expertos en Promoción de Salud y Educación. Con este Instrumento se ha comprobado si los currículos oficiales de la educación obligatoria publicados en el BOE y en el DOCV contribuyen adecuadamente a la adquisición de las competencias en Higiene. torsión en el aprendizaje introduciendo concepciones superadas por la comunidad científica.

Palabras clave: Higiene, Competencias en Salud, Currículo, Educación para la Salud, Salud escolar.

Abstract: The aim of this research has been to determine Hygiene competences that Spanish citizens must acquire in their compulsory step for the school and to make an approach to the competences in this topic that students can acquire through the possibilities provided in the Spanish official curricula. To achieve these objectives an analysis instrument has been defined. This instrument includes the nine main problems in Hygiene and the competences that students must acquire to face them. For this, a Delphi study and a meeting of experts in Health Promotion and Education have been carried out. Official curricula of Primary and Secondary Education, published in the Spanish Official Gazette (BOE) and the Official of the Autonomous Community of Valencia (DOCV), have been analyzed with the elaborated instrument in order to evaluate if they attend correctly to the Hygiene competences that students must acquire.

Keywords: Hygiene, Health competences, Curriculum, Health Education, Compulsory education.

(Fecha de recepción: junio, 2014, y de aceptación: septiembre, 2014)

DOI: 10.7203/DCES.28.4173 


\section{Introducción}

Existen conductas que con facilidad podemos calificar de higiénicas: lavarse las manos, el cuidado de los dientes, ducharse, etc. Parece que todo aquello relacionado al mantenimiento íntegro de la piel, de la barrera que delimita y protege nuestro cuerpo, forma parte de lo que denominamos Higiene.

También son fáciles de identificar como higiénicos algunos de los comportamientos relativos a la alimentación, como son la conservación y la manipulación de los alimentos; como también aquellas acciones que procuran evitar el contagio de enfermedades infecciosas: vacunarse, el uso del pañuelo ante los golpes de tos, el cuidado con los animales domésticos, etc.

Sin embargo, hay otros comportamientos que parecen menos relacionados con la higiene como el cuidado en la adopción de determinadas posturas corporales y movimientos, la prevención de enfermedades como puede ser el cáncer que, no obstante, requieren actuaciones que también podemos catalogar de higiénicas.

La importancia de este ámbito relativo a la salud es obvia, sin tratar de ser exhaustivos, basta recordar algunos datos:

-El cáncer es una de las primeras causas de muerte a nivel mundial. En 2012 se le atribuyeron nada menos que 8,2 millones de muertes. Aproximadamente un $30 \%$ de estas muertes se deben a cinco factores de riesgo comportamentales y alimentarios (índice de masa corporal elevado, consumo insuficiente de frutas y verduras, falta de actividad física y consumo de tabaco y alcohol) y, por lo tanto, pueden prevenirse.

-Las infecciones que pueden provocar cáncer, como las causadas por los virus de las hepatitis B y C y el del papiloma humano, son responsables del $20 \%$ de las muertes por cáncer en los países de ingresos bajos y medianos y del $7 \%$ en los países de ingresos altos. Se prevé que los casos anuales de cáncer aumentarán de 14 millones en 2012 a 22 en las próximas dos décadas.

-Los brotes de cólera siguen afectando a varios continentes durante 2013 y plantean un grave problema de salud pública en las poblaciones que no tienen acceso adecuado al agua potable y recursos sanitarios. -En los 21 países del "cinturón de la meningitis de África” (de Senegal a Etiopía) se han registrado más de 800000 casos en los últimos 15 años.

-Las infecciones de transmisión sexual (ITS) son una importante causa de enfermedad, infertilidad, discapacidad aguda y muerte. Cada año, 498 millones de personas se infectan con la clamidia, la gonorrea, la sífilis o la tricomoniasis.

-En 2012, se estima que 8,6 millones de personas desarrollaron la tuberculosis y 1,3 millones murieron por su causa. 
-El índice de parasitación intestinal entre los niños de 6 a 10 años en España es del 27,12\% (who, 2014; Pérez et al., 1997).

Todo esto nos señala la importancia que tienen las medidas higiénicas y la necesidad de que los ciudadanos las incorporen en sus comportamientos cotidianos. Sabedores de que el momento más adecuado para esta incorporación es el tiempo escolar ya que entonces es cuando se adquieren los hábitos y rutinas diarias, consideramos de especial importancia aproximarnos a la realidad escolar y estudiar el tratamiento que se le concede en la escuela a los temas de higiene.

Para llevar a cabo este estudio, debemos previamente concretar el significado de Higiene y el tipo de conductas relacionadas con ella que permitan hacer frente a los problemas derivados de su no observancia. Ello nos posibilitará determinar las competencias en Higiene que debe adquirir el alumnado en su paso por la enseñanza obligatoria y estudiar hasta qué punto se encuentran recogidas en el currículo escolar de la enseñanza obligatoria.

Así pues, los objetivos del presente trabajo son tres:

1. Determinar el significado de Higiene, diferenciándolo de la Promoción de la Salud y de la Salud Pública.

2. Concretar los problemas relativos a la Higiene más importantes que afectan a la población española, determinar las competencias en Higiene que se deben adquirir en la enseñanza obligatoria, y establecer los contenidos competenciales a desarrollar por el alumnado en relación a cada uno de los problemas de salud del ámbito de la Higiene.

3. Evaluar los currículos oficiales de la enseñanza obligatoria propuestos por el Ministerio de Educación, en su decreto de "enseñanzas mínimas" (BOE, 2006) y por la Generalitat Valenciana (DOCV) en relación a los contenidos competenciales definidos en el ámbito de la Higiene.

\section{Salud pública, Promoción de la Salud e Higiene}

A lo largo de la historia, salud y educación han estado unidas aunque de formas e intensidades distintas. Desde las civilizaciones más antiguas, la higiene se ha considerado un elemento importante en la vida de los pueblos y los gobernantes, de distintas épocas y sociedades, la han considerado como uno de los principales bienes sociales. Por dicho motivo y con la finalidad de llegar a todos los sectores de la población, surge la necesidad de difundir el conocimiento de la higiene a través de diversas vías de educación. Sin embargo, quizás sea conveniente concretar el concepto de Higiene y diferenciarlo de Salud pública, cuestión importante especialmente por lo tocante a la práctica educativa.

Si damos una mirada a la historia observamos cómo diversos pueblos desa- 
rrollaban medidas higiénicas dirigidas a preservar la salud de la población. Así, los egipcios poseían un sentido muy profundo de la limpieza personal, construían aljibes para almacenar el agua potable y canales para desaguar las aguas residuales. Los hebreos incluían en su propia ley la forma en que debían mantener el aseo de sus cuerpos, desinfectar las viviendas, aislar a los leprosos, eliminar las materias fecales, proteger el agua y los alimentos, procurar la máxima pulcritud a lo largo del tiempo de embarazo y de la maternidad, etc., de manera que se conoce al Levítico como el primer códice de higiene escrito (hacia el 1500 a.C.). La civilización griega desarrolló la higiene en un grado nunca alcanzado hasta ese momento, aunque la atención era casi exclusiva hacia el cuerpo: limpieza personal, ejercicio y alimentos. Los romanos construyeron acueductos para la conducción de agua potable a las ciudades, cloacas para evacuar los residuos líquidos y baños públicos en donde se prestaba gran atención a la limpieza corporal, preocupación ésta heredada de la cultura griega (Piédrola, 2008).

Durante la Edad Media se produce una reacción contraria a lo que significaba la cultura grecorromana. Los cuidados físicos pasaron a un segundo plano, como también todas aquellas cuestiones mundanas que no significaran una exaltación del espíritu. Las personas se bañaban poco, no les importaba llevar la ropa sucia, se acumulaban los desperdicios de las comidas en las casas y los excrementos y aguas residuales se echaban a la calle por las ventanas.
Estas actitudes y conductas, junto con los importantes movimientos migratorios por causas bélicas o religiosas (cruzadas, expansión del Islam, peregrinaciones, etc.), las bajas condiciones socioeconómicas y la carencia de alimentos, fueron las responsables de la rápida transmisión de una serie de enfermedades infecciosas importantes como la lepra o la peste bubónica (a mediados del siglo XIV, en algunos lugares de Europa, la peste -muerte negra- redujo la población a menos de la mitad). La lucha que la sociedad entabló contra ellas mediante la segregación de los leprosos, la cuarentena marítima y el establecimiento de cordones sanitarios, aunque terribles, son consideradas como las primeras medidas higiénicas de Salud pública, dirigidas expresamente a la protección de la salud mediante actividades organizadas y sistemáticas desde el Estado a través de la Administración.

No obstante, estas prácticas que podemos denominar "higiénicas", de las sociedades antiguas y medievales, no forman parte de una estrategia de Salud Pública como ahora lo entendemos, pese a encontrar en ellas elementos de protección de la salud por dos motivos: porque el concepto de sociedad no era como el actual, basta recordar el "estatus" de los esclavos o las dificultades para acceder a la condición de ciudadano romano; y porque todas las actividades iban dirigidas a los cuidados físicos de aquellos individuos que poseían determinados valores (económicos, jurídicos, atléticos, comerciales, 
etc.) y no para las personas enfermas, esclavas, inválidas o débiles.

La Salud Pública desde la perspectiva actual de filosofía social, práctica administrativa y política de gobierno, se desarrolla tan solo desde mediados del siglo XIX. Diversos autores (Kickbusch, 1986; Asthon y Seymour, 1988) señalan sus inicios hacia 1830, cuando Duncan, médico en Liverpool, se interesa por las condiciones de vivienda de los ciudadanos y descubre que más de un tercio de la población de las zonas industriales vive en condiciones infrahumanas, sin agua potable, ni alcantarillado y con una deficiente alimentación. En esta situación, las enfermedades florecen y se transmiten a gran velocidad, pues el contagio es muy fácil. El informe que redacta tuvo una gran repercusión. Chadwick en 1842 en Inglaterra y Shattuck en 1850 en Estados Unidos establecen con claridad la relación existente entre pobreza y enfermedad y demuestran que la miseria reinante en los barrios periféricos de las ciudades industriales, donde se instalan la suciedad, el desarraigo y la marginación, son la causa de la elevada tasa de mortalidad y baja esperanza de vida.

Con motivo de estos informes, los gobiernos de Europa y América del Norte crearon los primeros Servicios de Salud Pública bajo la denominación de "Sanidad e Higiene Pública", con el objeto de proteger la salud de la población mediante acciones de gobierno dirigidas al medio ambiente y a la colectividad. Se producen mejoras sociales y sanitarias: viviendas aceptables, apro- visionamiento de agua, construcción de colectores, lavaderos, casas de baños públicos, control sobre las adulteraciones de los alimentos, etc., que contribuyen a mejorar el estado de salud de la colectividad consiguiendo un gran descenso en la mortalidad (Piédrola, 2008).

Las acciones de estos servicios fueron sobre todo de vigilancia y control del medio ambiente y lucha contra las enfermedades transmisibles predominantes en esta época, mediante la legislación, la inspección, el saneamiento, la conducción de aguas potables y la verificación de los residuos. Consecuencia de esta etapa es el gran aumento de la población que Terris (1980) denomina "primera revolución epidemiológica". La reducción de la tasa de mortalidad es notable y las medidas que se toman consiguen una gran disminución de las enfermedades infecciosas (tuberculosis, gastroenteritis, bronquitis, neumonía, tos ferina, etc.). La actuación sobre el medio y los tratamientos preventivos lograron detener las grandes epidemias.

De esta forma, comenzamos a diferenciar los conceptos Higiene, Salud Pública y Promoción de Salud. Conviene señalar, sin embargo, la dificultad de definir el concepto de Higiene, atendiendo a la revisión bibliográfica realizada, ya que en algunos casos se confunde con el concepto de Salud Pública.

En el Diccionario Enciclopédico Ilustrado de Medicina (Dorland, 2003), se define Higiene como la "ciencia de la salud y su conservación" mientras que la Salud Pública se define como el "campo de la medicina que se ocupa de la aten- 
ción y el mejoramiento de la salud de la comunidad considerada globalmente".

Por su parte en el glosario de Promoción de la Salud de la OMS (1998), se define el término de Salud Pública como "la ciencia y el arte de promover la salud, prevenir la enfermedad y prolongar la vida mediante esfuerzos organizados de la sociedad".

En este sentido, con el objetivo de enmarcar la presente investigación, hemos optado, de acuerdo con Viñao (2010), por definir Higiene como el "conjunto de principios y prácticas tendentes a preservar la salud" y orientada "a mantener el cuerpo en buen estado físico y a evitar enfermedades".

Así mismo, siguiendo las recomendaciones de la OMS (1998) definimos Promoción de la Salud como "el proceso que permite a las personas incrementar el control sobre su salud para mejorarla" y Salud Pública como "la ciencia y el arte de promover la salud, prevenir la enfermedad y prolongar la vida”.

Así, la Salud Pública constituye una acción de gobierno y la Promoción de Salud una acción comunitaria, pero ambas encuentran en la Higiene un instrumento esencial para llevar a cabo parte de sus objetivos: mantener y preservar la salud.

\section{Competencias en Higiene a desarrollar en la educación obligatoria}

La Higiene forma parte de uno de los ámbitos de la salud, cuyas competencias ha estudiado el equipo de investigación
COMSAL (Competencias en Salud) constituido por 16 profesores de las Universidades de Valencia, Alicante, Elche y el País Vasco de diferentes áreas de conocimiento (Salud pública, Didáctica de las Ciencias Experimentales, Psicología, Pedagogía, Trabajo Social, Logopedia y Fisioterapia), varios profesores de Educación Primaria y Secundaria, y asesores de Centros de Profesores.

El estudio realizado ha consistido en una investigación cualitativa llevada a cabo en tres fases: 1- Identificación de los problemas/situaciones de salud que debe conocer el alumnado y propuesta de desarrollo de competencias; 2- Estudio Delphi para consensuar las propuestas de competencias para cada problema/ situación de salud; y 3- Reunión de expertos para consensuar los resultados obtenidos por el método Delphi.

Primera Fase: Para determinar los problemas o situaciones de salud a los que hay que atender en la enseñanza obligatoria se hizo una revisión de la literatura en la que aparece recomendaciones y propuestas que los organismos internacionales, nacionales y gobiernos autonómicos hacen sobre los problemas prioritarios en salud a fin de seleccionar aquellos más comunes. Para ello se buscó la información presente en las páginas web oficiales de estos organismos, así como en las publicaciones de los mismos en las que se citan y describen los problemas de salud de la población, haciendo especial énfasis en recabar los problemas relacionados con la salud de jóvenes y adolescentes. 
A partir de la revisión realizada se obtuvo un primer listado de problemas o situaciones prioritarios en salud existentes que debe conocer el alumnado. Dichos problemas se agruparon en "ámbitos de salud" teniendo como referencia la definición acordada por el equipo COMSAL como "las situaciones, contextos o entornos donde podemos encontrar un conjunto de problemas de salud relacionados entre sí, y sobre los que se puede actuar de forma conjunta" (Gavidia et al, 2012).

Así, se establecieron ocho ámbitos de salud: las Adicciones, la Alimentación y Actividad Física, la Sexualidad, la Salud Mental y Emocional, el Medio Ambiente, los Accidentes, la Promoción de la Salud y la Higiene, ámbito del que se ocupa el presente trabajo.

Posteriormente, el grupo investigador definió la competencia general de salud como "la capacidad y determinación de resolver los problemas relacionados con la salud personal y colectiva" o bien, y de una forma más desarrollada, como "la capacidad y el propósito de utilizar los recursos personales (habilidades, actitudes, conocimientos, experiencias, comportamientos...) para resolver de forma adecuada problemas de salud individual o colectivos en un contexto concreto y contribuir a crear un entorno en el que las opciones saludables sean fáciles de tomar". A continuación propuso las subcompetencias para cada uno de los ámbitos de salud a desarrollar en la enseñanza obligatoria, considerando en cada una de ellas sus tres dimensiones: saber (contenidos conceptuales), saber hacer (contenidos procedimentales) y saber estar y ser (contenidos actitudinales y/o conductuales), terminando con los contenidos competenciales necesarios para su adquisición.

Segunda Fase: Esta propuesta del grupo investigador se sometió a juicio de expertos externos siguiendo una metodología Delphi de dos rondas realizadas durante el año 2012 y se calificaron siguiendo una escala Likert de 5 puntos. Más tarde, los resultados obtenidos en la segunda ronda se valoraron en una reunión presencial de expertos, de forma que esta reunión sustituía una posible tercera ronda. La selección de los participantes se hizo atendiendo a su actividad profesional, formación académica y región de procedencia, confeccionando un panel heterogéneo (Gráfico 1), tratando de recoger, de esta forma, la diversidad de opiniones.

El primer envío del cuestionario se realizó a 113 personas expertas. En el segundo envío se aumentó la muestra a 160 expertos, tratando de conseguir al menos 10 opiniones externas por cada ámbito de salud, basándonos en las recomendaciones de Okoli y Pawlowski (2004). En el caso de la Higiene fueron 13 los expertos que llegaron a la segunda ronda Delphi.

Para el análisis de los resultados se calculó la Media (M) y la Desviación Típica (DT) obtenida en cada ítem, determinando de esta forma la importancia concedida y el grado de consenso suscitado en cada uno de ellos.

Tercera Fase: Con los resultados obtenidos se organizó la Reunión de 


\section{GRÁFICO 1. Caracterización de los expertos que intervienen en el estudio Delphi según procedencia geográfica y actividad profesional}

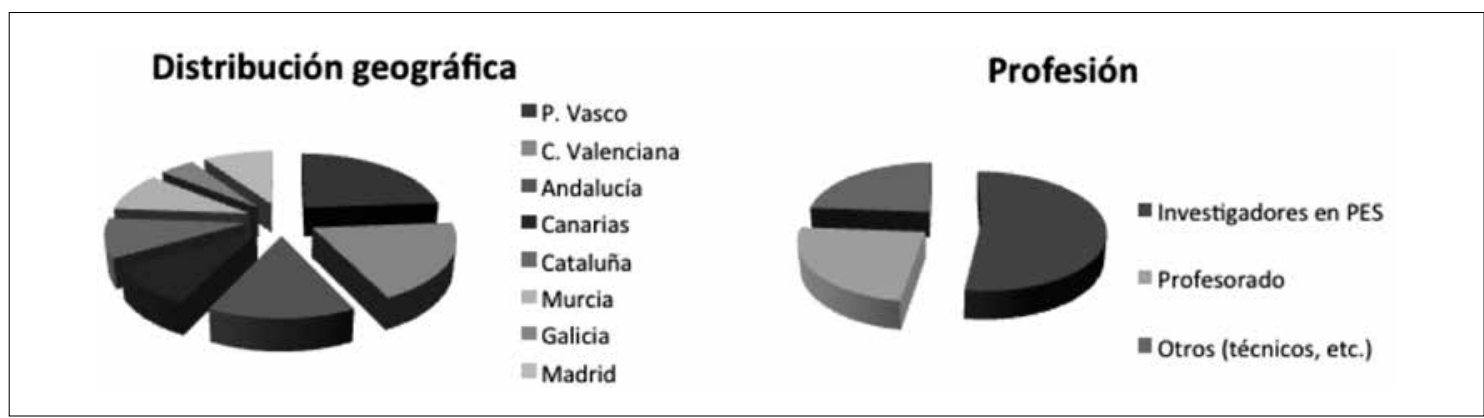

Expertos en Promoción y Educación para la Salud (marzo, 2013) en la Facultad de Magisterio de la Universitat de València. Los invitados a intervenir en esta jornada fueron los participantes en alguna de las dos rondas Delphi. En esta reunión los asistentes debatieron, junto con los miembros del equipo de investigación COMSAL, los datos de los cuestionarios hasta llegar a consensos en sus enunciados y valoraciones generales. Se estudiaron todos los ítems atendiendo las sugerencias de Coll y Martín de separar lo imprescindible o necesario de lo deseable ya que "no se puede enseñar todo lo que nos gustaría que los niños y jóvenes aprendiesen; ni siquiera lo que con toda seguridad es beneficioso que los niños y jóvenes aprendan”.

De esta forma, las competencias y los contenidos competenciales necesarios para su desarrollo fueron valorados teniendo en cuenta la puntuación otorgada a cada ítem ( $1=$ muy en desacuerdo y $5=$ muy de acuerdo) y la Desviación Típica. La valoración usó las siglas SIP, que tuvo en cuenta los criterios siguientes: $4 \leq \mathrm{M}-\mathrm{DS} \leq 5=\mathrm{S}$ (Sustancial o Necesario), $3 \leq \mathrm{M}-\mathrm{DS}<4=\mathrm{I}$ (Importante) y $\mathrm{M}-\mathrm{DS}<3=\mathrm{P}$ (Prescindible). La Reunión de Expertos concluyó con el diseño definitivo del Instrumento de análisis del ámbito de la Higiene y de los demás ámbitos de salud.

Los temas relacionados con la Higiene consensuados para ser abordados en durante la educación obligatoria fueron: higiene bucodental, higiene corporal, enfermedades infecciosas, parasitismo, alergias, cáncer, higiene postural, higiene sexual e higiene de los alimentos.

En el Anexo se presentan las competencias de Higiene para hacer frente a los problemas mencionados y los contenidos competenciales necesarios para su desarrollo en la escuela. En negrita están los contenidos considerados necesarios o sustanciales y en tipo de letra normal los contenidos consideras como importantes. Aquellos contenidos que fueron considerados como prescindibles no aparecen. 


\section{Análisis de los currículos de la enseñanza obligatoria}

Con los contenidos competenciales en Higiene se conforma el Instrumento de análisis con el que abordar las propuestas oficiales de los currículos y comprobar hasta qué punto las distintas administraciones han tenido en cuenta esta problemática en su elaboración. De esta forma, para atender al tercero de los objetivos previstos, se ha utilizado como muestra los currículos prescriptivos de la Ley Orgánica de Educación (LOE) que aparecen en el BOE (2006) como enseñanzas mínimas en Educación Primaria y Educación Secundaria Obligatoria, y los decretos curriculares que establece el Gobierno Valenciano a través del DOCV (2007).

Para llevar a cabo el análisis curricular, se procedió a la lectura de los currículos y se fueron anotando aquellos elementos curriculares (objetivos, contenidos y criterios de evaluación) que hacen referencia a los distintos contenidos competenciales definidos en el Instrumento de análisis. Dicho análisis fue consensuado entre dos personas del grupo de investigación y sus resultados revisados por otro investigador. Posteriormente, se efectuó el recuento dichas aportaciones curriculares. A continuación se muestran los resultados obtenidos.

\section{GRÁFICO 2. Contribución curricular al desarrollo de las competencias en}

Higiene de los currículos oficiales analizados (BOE y DOCV) por asignaturas

\section{Contribución curricular al desarrollo de las competencias en Higiene}

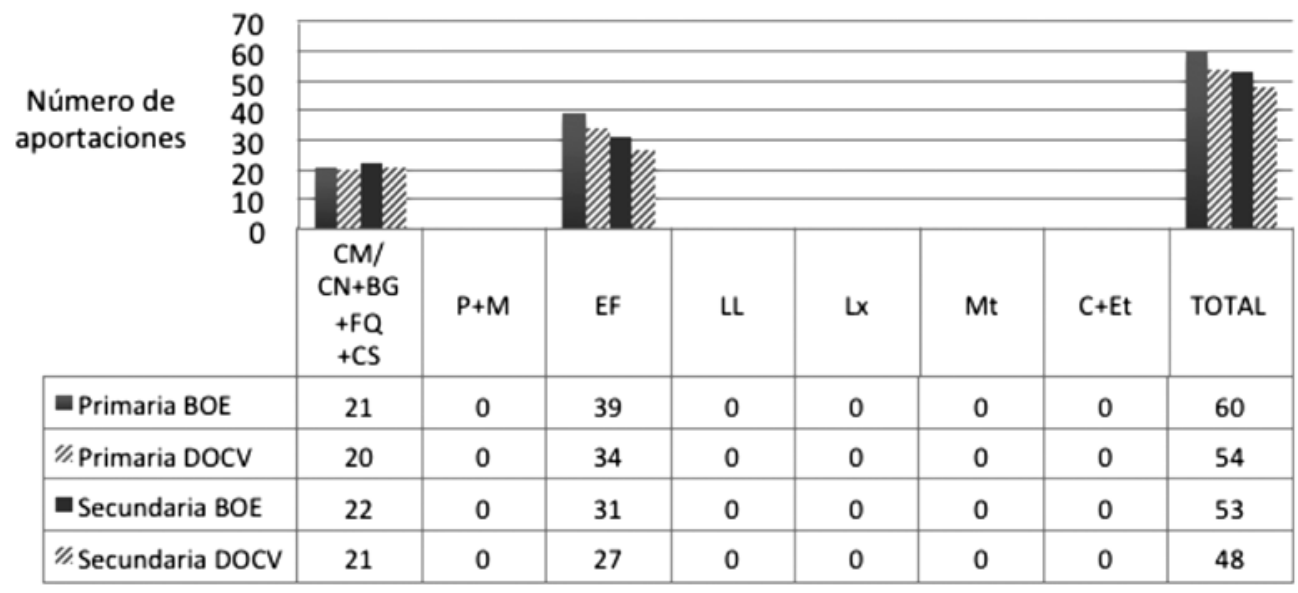

Nota: CM: Conocimiento del Medio; CN: Ciencias Naturales; BG: Biología y Geología; P: Plástica; M: Música; EF: Educación Física; LL: Lengua Castellana y cooficial; Lx: Lengua extranjera; Mt: Matemáticas, C: Ciudadanía; Et: Ética. 
Atendiendo al número de aportaciones curriculares, se observa que en los currículos prescriptivos de la educación obligatoria publicados en el $\mathrm{BOE}$ (113) se contribuye en mayor medida a la adquisición de las competencias en Higiene que en los publicados en el DOCV (102). Además, la contribución curricular en la Educación Primaria (60 en el BOE y 54 en el DOCV) es mayor a la de la Educación Secundaria Obligatoria (53 en el BOE y 48 en el DOCV). Las materias y/o áreas de conocimiento que más contribuyen al desarrollo de las competencias en Higiene son: Conocimiento del Medio Natural, Social y Cultural (y las materias equivalentes en ESO) y Educación Física.

Si observamos la contribución por materias y/o áreas de conocimiento se observa el elevado número de aportaciones curriculares de la materias y áreas de conocimiento de Educación Física (70 en el BOE y 61 en el DOCV) y de Conocimiento del Medio Natural, Social y Cultural y sus materias equivalentes en la ESO (43 en el BOE y 41 en el DOCV) y, por tanto, su importancia en la adquisición de las competencias en Higiene. No obstante, se encontraron también algunos elementos curriculares en las materias y/o áreas de la Educación Artística (y sus materias equivalentes en la ESO) y de la Educación para la Ciudadanía y los Derechos Humanos que, aunque no hacen referencia explícita a los contenidos competenciales del Instrumento de análisis, dependiendo de la sensibilidad pedagógica del profesorado, pueden también contribuir a mejorar las com- petencias en higiene del alumnado de la educación obligatoria.

El gráfico 3 muestra las dimensiones (conocimientos, procedimientos y actitudes) que se encuentran más representadas en el tratamiento de la Higiene por los currículos oficiales (BOE y DOCV). Aunque las tres dimensiones se encuentran representadas, la que más aparece en los currículos oficiales es la de los conocimientos seguida de las actitudes y, por último, los procedimientos. Únicamente, en el currículo de la ESO (BOE) encontramos una excepción ya que aparece mayor número de aportaciones en la dimensión de los procedimientos que en el de las actitudes.

A continuación, se ha desglosado por dimensiones las aportaciones curriculares del Conocimiento del Medio y de la Educación Física que son las asignaturas que más contribuyen a la adquisición de las competencias en Higiene, tanto en Educación Primaria como en Secundaria. Se ha optado por mostrar solamente los resultados obtenidos en el análisis de los currículos oficiales publicado en el BOE. los resultados en el DOCV son muy similares. (Gráfico 4)

Observando el gráfico 4, comprobamos que la Educación Física en Primaria, la dimensión actitudinal es la más representada seguida de los conocimientos y, por último, de los procedimientos. Sin embargo, en la ESO encontramos un mayor número de aportaciones curriculares que atiendan a la dimensión de los conocimientos, seguida de la dimensión procedimental y, por último, la actitudinal. 
GRÁFICO 3. Número de aportaciones curriculares por dimensiones que contribuyen al desarrollo de las competencias en Higiene (BOE y DOCV)

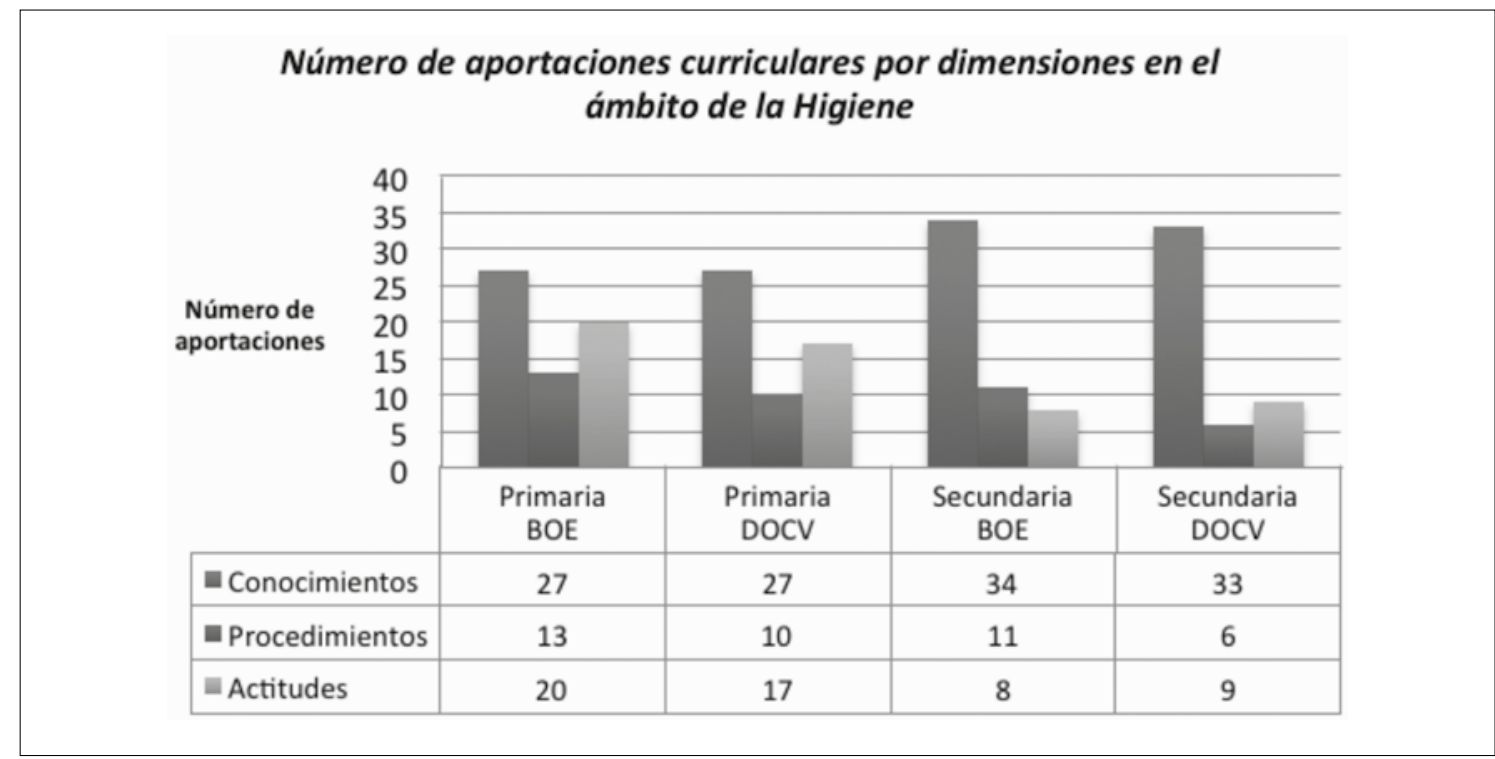

GRÁFICO 4. Número de aportaciones curriculares de C. del Medio y Ed Física al desarrollo de las competencias en Higiene en Ed. Primaria y en Secundaria (BOE)

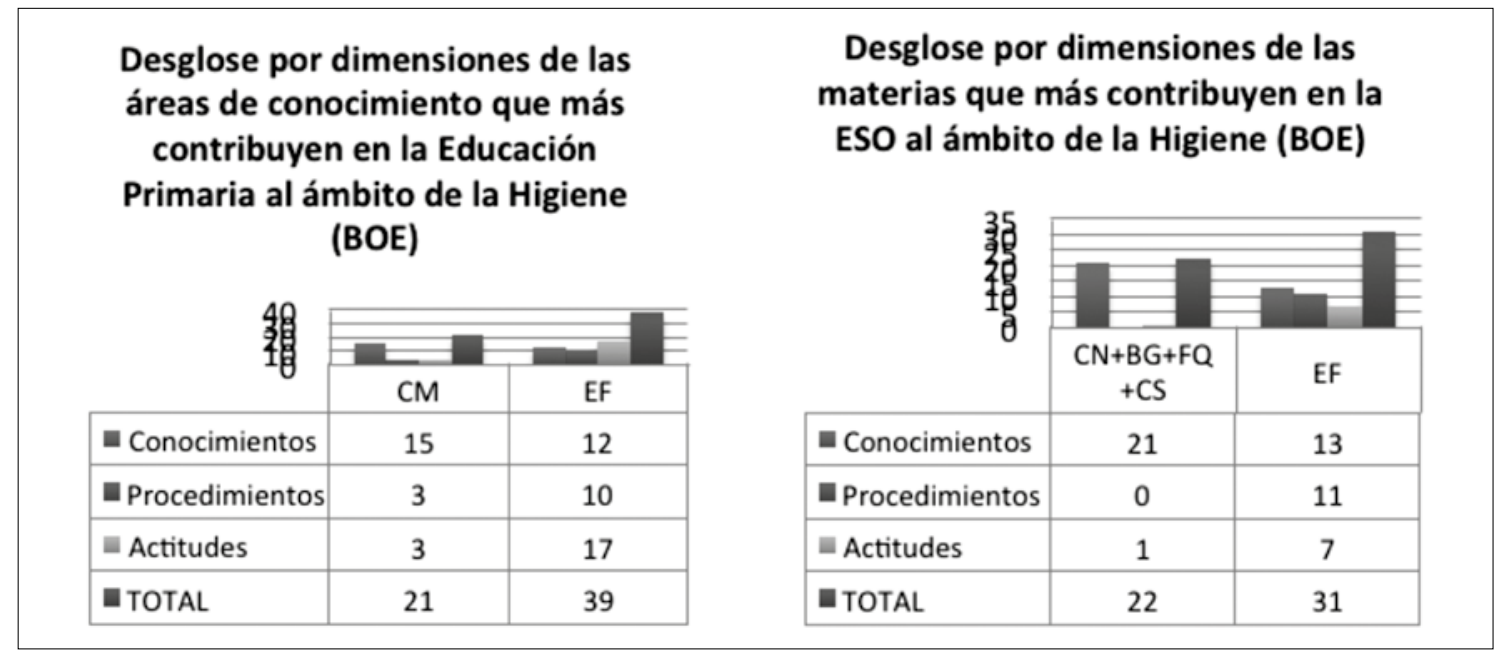

Si se analiza el área de Conocimiento del Medio Natural, Social y Cultural (y sus materias equivalentes en la
ESO) se observa que en la Educación Primaria las aportaciones relativas a los conocimientos son bastante superio- 
res. En el caso de la ESO, cabe destacar que la contribución a la dimensión procedimental es inexistente y solamente se observa una única aportación curricular de tipo actitudinal. Es decir, la contribución curricular desde el área de Conocimiento del Medio Natural, Social y Cultural (y sus materias equivalentes en la ESO) se centra casi exclusivamente en los conocimientos de contenidos.

Seguidamente, la tabla 1 muestra el número de aportaciones curriculares (BOE y DOCV) que recibe cada uno de los nueve problemas o situaciones problemáticas relacionadas con el ámbito de salud de la Higiene. En ella se observa que, en los currículos oficiales de la educación obligatoria (BOE y DOCV), no aparecen representados todos los problemas de salud o situaciones problemáticas que conforman el ámbito de la Higiene. Así, no aparece referencia alguna a los contenidos competenciales para tratar la prevención del cáncer, ni para abordar la higiene de los alimentos y solo una única aportación curricular (tanto en el BOE como en el DOCV) hace mención al problema de las alergias.

Por otro lado, las situaciones problemáticas del parasitismo, la higiene bucodental, las enfermedades infecciosas y la higiene sexual aparecen recogidas en los currículos aunque con pocas aportaciones.

Los problemas de Higiene más representados en los currículos (BOE

\section{TABLA 1. Número de aportaciones curriculares a los diferentes problemas de salud del ámbito de la Higiene (BOE y DOCV)}

\begin{tabular}{|lcc|}
\hline $\begin{array}{l}\text { Aportaciones curriculares en la } \\
\text { educación obligatoria }\end{array}$ & $\begin{array}{c}\text { Número de } \\
\text { aportaciones (BOE) }\end{array}$ & $\begin{array}{c}\text { Número de } \\
\text { aportaciones(DOCV) }\end{array}$ \\
\hline P.1 Ante la higiene bucodental & 5 & 4 \\
\hline P.2 Ante la higiene corporal & 24 & 19 \\
\hline P.3 Ante las enfermedades infecciosas & 7 & 6 \\
\hline P.4 Ante el parasitismo & 3 & 3 \\
\hline P.5 Ante las alergias & 1 & 1 \\
\hline P.6 Para la prevención del cáncer & 0 & 0 \\
\hline P.7 Ante la higiene postural & 65 & 61 \\
\hline P.8 Ante la higiene sexual & 8 & 8 \\
\hline P.9 Ante la higiene de los alimentos & 0 & 0 \\
\hline TOTAL & 113 & 102 \\
\hline
\end{tabular}


y DOCV) son la higiene postural y la higiene corporal. El primero de ellos cuenta con más de la mitad de número de aportaciones que contribuyen al desarrollo de las competencias en Higiene (65 de 113 en el BOE y 61 de 102 en el DOCV).

\section{TABLA 2. Contenidos competenciales de Higiene que no aparecen en los currículos oficiales (BOE)}

\section{Conocimientos ausentes}

- El peligro de los piercings y tatuajes.

- Principales parásitos que afectan al ser humano, por ejemplo lombrices, tenias y piojos. Reservorios y vías de entrada del parásito.

- Concepto de alergia. Principales alérgenos (pelos, alimentos, polvo, etc.). Síntomas que producen las alergias.

- Concepto de cáncer.

- Concepto de higiene alimentaria. Medidas básicas ante posibles infecciones e intoxicaciones alimentarias.

\section{Procedimientos ausentes}

- Reconocer los síntomas de una infección.

- Identificar las medidas de salud pública que permiten aminorar la transmisión de enfermedades infecciosas.

- Saber aplicar las medidas higiénicas apropiadas en la relación con animales domésticos.

- Relacionar las épocas estacionales con la aparición de las alergias más comunes.

- Identificar los alérgenos que pueden aparecer en las etiquetas, los menús, la bolsa de la compra, etc.

- Practicar autoexámenes de los testículos y autoexploración mamaria regularmente para el diagnóstico precoz de enfermedades.

- Reconocer la aparición de síntomas ligados a infecciones (olores fuertes, picazón o ardor).

- Reconocer los síntomas ante posibles infecciones e intoxicaciones alimentarias (náuseas, dolor abdominal, vómitos, diarrea, fiebre, etc.).

- Reconocer alimentos en mal estado.

\section{Actitudes ausentes}

- Tomar conciencia de las repercusiones de una mala higiene bucodental.

- Reconocer en el uso adecuado de la ropa, un elemento que favorece la aceptación de los demás y la de uno mismo.

- Tomar conciencia de la repercusión de las enfermedades infecciosas y su transmisión.

- Valorar las medidas de salud pública y acudir a los servicios médicos cuando sea necesario.

- Estar alerta ante las propias respuestas y las del entorno ante posibles alérgenos.

- Tomar conciencia de la importancia de realizar revisiones periódicas para la posible identificación temprana del cáncer y acudir a los servicios médicos cuando sea necesario.

- Tomar conciencia de la importancia de realizar revisiones periódicas y acudir a los servicios sociosanitarios ante cualquier duda.

- Tomar conciencia de la importancia de la fecha de caducidad y consumo preferente de los alimentos. 
Los contenidos competenciales que no se desarrollan en los currículos son prácticamente los mismos en el $\mathrm{BOE}$ que en el DOCV. Entre las ausencias destacan conceptos tan básicos como parasitismo, alergia y cáncer. Por otra parte, también resulta llamativa la ausencia de aportaciones curriculares referentes a reconocer los síntomas de las distintas enfermedades. Es decir, aparecen contenidos curriculares relativos a las distintas enfermedades (principalmente la enfermedad como concepto y los tipos de enfermedad), sin embargo, no aparece el reconocimiento o identificación de síntomas como las náuseas, el dolor abdominal, los vómitos, la fiebre, etc. Por último, también llamar la atención sobre la ausencia de aportaciones en los currículos referentes a la realización de revisiones periódicas como medio de prevención y a utilizar los recursos sanitarios como vía de información y de prevención (Tabla 2).

\section{Conclusiones}

El presente estudio ha permitido concretar la competencia en Higiene necesaria a desarrollar durante la etapa escolar de la educación obligatoria; las dimensiones del saber, saber hacer y saber estar y ser que integran esta competencia; los problemas o situaciones problemáticas relacionadas con la Higiene que afectan a los jóvenes españoles; $y$, finalmente, los contenidos competenciales necesarios para hacerles frente. Con todo ello se ha elaborado un instrumento de análisis curricular para este ámbito y se ha aplicado a los currículos oficiales de la educación obligatoria publicados en el BOE y en el DOCV.

De los resultados obtenidos del análisis curricular se concluye que tanto en la Educación Primaria como en la Secundaria existen un buen número de aportaciones curriculares, que permiten desarrollar las competencias en Higiene, pero las de Primaria son superiores que las de la ESO, en ambos currículos (BOE y DOCV). No obstante, las publicadas en el BOE, que establecen las "enseñanzas mínimas", contribuyen en mayor medida que las publicadas en el DOCV, en las que sería de esperar una mayor concreción y desarrollo.

Las materias o áreas de conocimiento que más intervienen en la adquisición de la citada competencia son la Educación Física y el Conocimiento del Medio Natural, Social y Cultural y su equivalente en la ESO de Ciencias de la Naturaleza. Aunque la aportación del resto de las áreas de conocimiento y materias es nula, dependiendo de la sensibilidad pedagógica del profesorado, se pueden encontrar contenidos que contribuyan a mejorar las competencias en la higiene del alumnado.

En la etapa de Educación Primaria, la dimensión de los conocimientos es la más representada en los currículos oficiales (BOE y DOCV), seguida de las actitudes y, por último, los procedimientos. En la Educación Secundaria Obligatoria también los conocimientos es la dimensión más contemplada. En el 
BOE es seguida de los procedimientos y en el DOCV de las actitudes.

El problema o situación problemática del ámbito de la Higiene que es tratada con mayor consideración en los currículos analizados es la higiene postural, con más de la mitad del número total de aportaciones curriculares. En segundo lugar, se encuentra la higiene corporal. Los problemas relativos a la higiene sexual, las enfermedades infecciosas, la higiene bucodental y el parasitismo también se contemplan en los currículos aunque reciben un menor número de aportaciones. Las alergias apenas se contemplan y la prevención del cáncer y la higiene de los alimentos no tienen representación curricular.

Algunos de los contenidos competenciales definidos en el Instrumento de análisis no aparecen representados en los currículos oficiales analizados. Entre las ausencias, destacan conceptos como el cáncer y la alergia, y procedimientos como el reconocimiento de los síntomas de las distintas enfermedades, la realización de revisiones periódicas y la utilización de los recursos sanitarios como vía de información y de prevención.

Finalmente, se considera que el Instrumento de análisis que se propone en el presente trabajo puede contribuir al desarrollo de las competencias en Higiene en la educación obligatoria, y puede servir de referencia para elaborar material didáctico por el profesorado e instituciones educativas, libros de texto por las editoriales y útil para la concreción de propuestas curriculares por las distintas administraciones.

\section{Referencias bibliográficas}

ASTHON, J. y SEYMOUR, H. (1988). La nueva Salud Pública. Masson: Barcelona.

CHAWICK, H.D. (1937). The diseases of the inhabitants of the Commonwealth. N.Engl. J.Med., 216, 8.

COLL C, y MARTÍN E. (2006). Vigencia del debate curricular. Aprendizajes básicos, competencias y estándares. PRELAC-UNESCO 3: pp. 6-27

DECRETO 111/2007, de 20 de julio, del Consell, por el que se establece el currículo de la Educación Primaria en la Comunitat Valenciana. DOCV, 24 de julio de 2007.

DECRETO 112/2007, de 20 de julio, del Consell, por el que se establece el currículo de la Educación Secundaria Obligatoria en la Comunitat Valenciana. DOCV, 24 de julio de 2007.

DORLAND (2003). Diccionario Enciclopédico Ilustrado de Medicina. Madrid: McGraw-Hill Interamericana.

GAVIDIA, V, TALAVERA, M, SENDRA, C. LLORENTE, E GARCÍA DE LA GERA, M y GOMAR, B. (2012) The evaluation of competences in health. Proceedings of INTED2012. Conference, pp: 166-173.

KICKBUSCH, I. 1986. Health promotion strategies for action. Canadian Journal of Public Health, 77 (5), 321326.

LEY ORGÁNICA 2/2006, de 3 de mayo, de Educación (LOE). BOE, 4 de mayo de 2006.

OKOLI, C. y PAWLOWSKI, S.D. (2004). The Delphi method as a research 
tool: an example, design considerations and applications. Information and Management, 42 (1), 5-29.

ORGANIZACIÓN MUNDIAL DE LA SALUD (1998). Promoción de la Salud. Glosario. Ministerio de Sanidad y Consumo.

ORGANIZACIÓN MUNDIAL DE LA SALUD (2014). Información sobre el cáncer. En http://www.who.int/cancer/about/facts/es/ [consultado en julio, 2014].

PÉREZ, C., ARIZA, C., ÚBEDA, J.M., GUEVARA, D.C., DE ROJAS, M. y LOZANO, C. (1997). Epidemiología del parasitismo intestinal en el valle del Guadalquivir, España. Revista Española de Salud Pública, 71, 547552.

PIÉDROLA, G. (2008). Medicina preventiva y salud pública. $11^{\mathrm{a}}$ ed. Barcelona: Elsevier Masson.
REAL DECRETO 1513/2006, de 7 de diciembre, por el que se establecen las enseñanzas mínimas correspondientes a la Educación Primaria. BOE, 8 de diciembre de 2006

REAL DECRETO 1631/2006, de 29 de diciembre, por el que se establecen las enseñanzas mínimas correspondientes a la Educación Secundaria Obligatoria. BOE, 5 de enero de 2007. SHATTUCK, L. et al. (1948). Report of the Sanitary Commission of Massachussets (Dutton and Wentworth, State Printers, Boston, 1850).:Cambridge (Massachussets): Harvard University Press.

TERRIS, M. (1980). La revolución epidemiológica y la medicina social. México: siglo XXI.

VIÑAO, A. (2010). Higiene, salud y educación en su perspectiva histórica. Educar, 36, 181-213. 


\section{ANEXO. Instrumento de análisis curricular en el ámbito de la Higiene.}

Competencia: "Desarrollar y mantener pautas de higiene diaria que prevengan la aparición de enfermedades y mejoren la calidad de vida".

Saber: Las medidas necesarias para el cuidado del cuerpo y las consecuencias de no hacerlo. Los agentes que pueden producir enfermedades y sus vías de transmisión.

Saber hacer: Desarrollar las pautas de conducta necesarias para mantener una higiene corporal y ambiental correcta. Utilización adecuada de los servicios médicos.

Saber ser: Evitar ser un agente vector en la transmisión de enfermedades. Desarrollar estrategias para mejorar la salud personal y ambiental.

\section{Situación problemática: Ante la Higiene Bucodental}

\section{Conocimientos a adquirir}

- Anatomía y funciones de las estructuras bucodentales.

- Enfermedades relacionadas con problemas bucodentales (caries, gingivitis, etc.).

- Factores de prevención. Higiene y cuidados de la lengua, encías y dientes.

- Influencia de la alimentación en el mantenimiento de una boca sana.

\section{Procedimientos a utilizar}

- Identificar los elementos que causan daño a la salud bucodental (mala nutrición, tabaco, ausencia de un correcto cepillado, etc.)

- Hacer buen uso de los utensilios destinados al cuidado e higiene de la boca (cepillos, seda dental, etc.).

\section{Actitudes a desarrollar}

- Adquisición de los hábitos necesarios para mantener una boca sana.

- Generar hábitos dietéticos adecuados que eviten el exceso de productos azucarados.

- Apreciar la necesidad de acudir al dentista de manera regular.

- Tomar conciencia de las repercusiones de una mala higiene bucodental.

\section{Situación problemática: Ante la Higiene Corporal}

\section{Conocimientos a adquirir}

- Anatomía y fisiología de la piel y de los órganos de los sentidos.

- Normas higiénicas para mantener una vida saludable (limpieza corporal, de la ropa, calzado, etc.).

- El peligro de los piercings y tatuajes.

- Consecuencias de no mantener una correcta higiene diaria.

\section{Procedimientos a utilizar}

- Identificar las características de las distintas prendas de vestir con su uso adecuado y su relación con las estaciones del año.

- Saber llevar a cabo las prácticas de higiene corporal.

\section{Actitudes a desarrollar}

- Realización de una correcta higiene corporal.

- Tomar conciencia de los riesgos de no tener hábitos de higiene.

- Reconocer en el uso adecuado de la ropa, un elemento que favorece la aceptación de los demás y la de uno mismo. 


\section{Situación problemática: Ante las Enfermedades Infecciosas}

\section{Conocimientos a adquirir}

- Concepto de infección, sus fuentes y mecanismos de transmisión. Principales enf. infecciosas actuales.

- Medidas preventivas para evitar el contagio. Ejemplo: la vacunación.

- Funcionamiento básico del sistema inmunológico.

\section{Procedimientos a utilizar}

- Reconocer los síntomas de una infección

- Identificar las medidas de salud pública que permiten aminorar la transmisión de enf. infecciosas.

- Analizar la prevalencia e incidencia de enfermedades infecciosas en diferentes países.

\section{Actitudes a desarrollar}

- Tomar conciencia de la repercusión de las enfermedades infecciosas y su transmisión.

- Desarrollar conductas responsables que eviten la transmisión de enfermedades.

- Valorar las medidas de salud pública y acudir a los servicios médicos cuando sea necesario.

- Sensibilidad y solidaridad ante enfermedades de especial incidencia en países en vías de desarrollo.

\section{Situación problemática: Ante el Parasitismo}

\section{Conocimientos a adquirir}

- Concepto de parasitismo. Principales parásitos que afectan al ser humano, p.e. lombrices, tenias y piojos. - Reservorios y vías de entrada del parásito.

- Medidas de higiene que evitan contagios.

\section{Procedimientos a utilizar}

- Identificar las medidas de higiene que permiten prevenir el contagio de parásitos (lavado de manos, cabellos, preparación de alimentos, etc.).

- Saber aplicar las medidas higiénicas apropiadas en la relación con animales domésticos.

\section{Actitudes a desarrollar}

- Desarrollar conductas responsables frente a posibles contagios

- Introducir en la vida cotidiana personal y ambiental las medidas de higiene adecuadas.

\section{Situación problemática: Ante las alergias}

\section{Conocimientos a adquirir}

- Concepto de alergia. Principales alérgenos (pelos, alimentos, polvo, etc.).

- Síntomas que producen las alergias

\section{Procedimientos a utilizar}

- Saber aplicar medidas de higiene para minimizar los efectos de las alergias.

- Relacionar las épocas estacionales con la aparición de las alergias más comunes.

- Identificar los ingr. que pueden producir las alergias en las etiquetas, los menús, la bolsa de la compra...

\section{Actitudes a desarrollar}

- Predisposición a realizar labores de limpieza en el hogar que contribuyan a la higiene ambiental.

- Estar alerta ante las propias respuestas y las del entorno ante posibles alérgenos. 


\section{Situación problemática: Para la prevención del cáncer}

\section{Conocimientos a adquirir}

- Concepto de cáncer.

- Factores de riesgo: genética, medio ambiente, estilos de vida.

- Medidas de prevención y detección de cáncer.

\section{Procedimientos a utilizar}

- Reconocer las pautas de comport. apropiadas para minimizar los riesgos de la aparición del cáncer. - Analizar los principales factores ambientales presentes en el entorno próximo que puedan inducir a la aparición de cáncer.

\section{Actitudes a desarrollar}

- Adoptar estilos de vida saludables que pueda prevenir el cáncer.

- Tomar conciencia de la importancia de realizar revisiones periódicas para la posible identificación temprana del cáncer y acudir a los servicios médicos cuando sea necesario.

\section{Situación problemática: Ante la Higiene Postural}

\section{Conocimientos a adquirir}

- Anatomía y fisiología del aparato locomotor (sistemas esquelético y muscular).

- Enfermedades relacionadas con el aparato locomotor (desviaciones de la columna vertebral, hernias, esguinces, roturas, etc.).

- Medidas de prevención. Hábitos posturales adecuados y actividad física.

\section{Procedimientos a utilizar}

- Identificar los elementos que inciden negativamente en el aparato locomotor (adopción de malas posturas, realización de movimientos incorrectos, sedentarismo, obesidad, etc.)

- Reconocer y utilizar las medidas adecuadas para evitar la aparición de patologías relacionadas con el aparato locomotor.

\section{Actitudes a desarrollar}

- Adquisición de los hábitos posturales adecuados, tomando conciencia de las repercusiones de una mala higiene postural.

- Valorar los beneficios derivados de la realización de actividad física. 


\section{Situación problemática: Ante la Higiene Sexual}

\section{Conocimientos a adquirir}

- Anatomía y fisiología de los aparatos genitales femenino y masculino.

- Medidas de higiene sexual.

- Las ETS, vías de contagio y sus consecuencias para la salud.

- Métodos de protección frente a las ETS.

\section{Procedimientos a utilizar}

- Practicar autoexámenes de los testículos después del baño y la autoexploración mamaria regularmente para el diagnóstico precoz de enfermedades.

- Utilizar las medidas necesarias para llevar a cabo una adecuada higiene genital diaria (ducha diaria así como lavar y secar bien los genitales).

- Reconocer la aparición de síntomas ligados a infecciones (olores fuertes, picazón o ardor).

- Utilizar los métodos de protección necesarios frente a las ETS.

\section{Actitudes a desarrollar}

- Tomar conciencia de la importancia de realizar revisiones periódicas y acudir a los servicios sociosanitarios ante cualquier duda.

- Tomar conciencia de la importancia de llevar a cabo una adecuada higiene genital para evitar infecciones.

- Tomar conciencia de los riesgos para la salud que conllevan las relaciones sexuales no seguras.

\section{Situación problemática: Ante la Higiene de los alimentos}

\section{Conocimientos a adquirir}

- Concepto de higiene alimentaria.

- Principales Enfermedades de Transmisión Alimentaria. Concepto de infección e intoxicación alimentaria.

- Métodos de prevención de las Enfermedades de Transmisión Alimentaria.

- Medidas básicas ante posibles infecciones e intoxicaciones alimentarias.

\section{Procedimientos a utilizar}

- Identificar los factores que producen enfermedades derivadas del tratamiento de los alimentos (temperatura inadecuada en la conservación, falta de limpieza, cocción insuficiente, manipulaciones incorrectas, etc.)

- Reconocer los síntomas ante posibles infecciones e intoxicaciones alimentarias (náuseas, dolor abdominal, vómitos, diarrea, fiebre, etc.)

- Reconocer alimentos en mal estado.

- Llevar a cabo medidas adecuadas de higiene en la conservación y manipulación de los alimentos.

\section{Actitudes a desarrollar}

- Valorar la necesidad de poner en práctica las normas básicas de higiene alimentaria.

- Tomar conciencia de la importancia de la fecha de caducidad y consumo preferente de los alimentos.

- Introducir en la vida diaria hábitos de una correcta conservación y manipulación de los alimentos. 